\title{
Brownian Diffusivities of Interacting Colloidal Particles Measured by Dynamic Light Scattering
}

\author{
Aernout van Veluwen, Hendrik N. W. Lekkerkerker, ${ }^{*}$ Cornelus G. de Kruif \\ and Agienus Vrij \\ Van 't Hoff Laboratory, University of Utrecht, Padualaan 8, \\ $3584 \mathrm{CH}$ Utrecht, The Netherlands
}

\begin{abstract}
In a system of interacting colloidal particles one can distinguish collective diffusion, short-time and long-time self-diffusion and exchange diffusion. The meanings of and connections between these diffusivities are established. It is shown how these various Brownian diffusivities may be obtained from dynamic light scattering measurements on monodisperse and polydisperse systems. Experimental results for both dilute and concentrated sterically stabilized silica dispersions are presented. The experimental concentration dependence of the various diffusivities is compared with theoretical predictions.
\end{abstract}

\section{Introduction}

With the large increase of activity in the study of concentrated colloidal dispersions in recent years there has arisen a considerable interest in the Brownian motion of interacting colloidal particles. ${ }^{1,2}$ These motions are influenced by direct interactions and by hydrodynamic interactions, both of which depend on the spatial configuration of the particles. This makes the calculation of the Brownian diffusivity of interacting colloidal particles a challenging many-body problem in statistical mechanics and hydrodynamics. ${ }^{3}$

For independent spherical colloidal particles the Brownian motion can be characterized by the single-particle diffusion coefficient, which is given by the well known Stokes-Einstein expression

$$
D_{0}=\frac{k T}{6 \pi \eta a}
$$

where $\boldsymbol{k}$ is Boltzmann's constant, $T$ is the absolute temperature, $\eta$ is the viscosity of the Newtonian solvent and $a$ is the radius of the particle. ${ }^{4}$ In the limit of infinite dilution the quantity $D_{0}$ determines both collective diffusion as well as self-diffusion.

Collective diffusion refers to the process by which concentration gradients gradually disappear owing to the Brownian movement of the particles. This process can be described by the diffusion equation

$$
\frac{\partial \rho}{\partial t}=D_{\mathrm{c}} \nabla^{2} \rho
$$

where $\rho$ is the number density of the colloidal particles and $D_{\mathrm{c}}$ is the collective diffusion coefficient.

Self-diffusion refers to the Brownian motion of a particle in a suspension of uniform concentration. A quantitative measure of self-diffusion is the mean-square displacement $\left\langle\Delta r^{2}(t)\right\rangle$ of this particle as a function of time. For non-interacting colloidal particles the mean-square displacement is linear in time for times larger than the relaxation time $\tau_{B}$ of the Brownian fluctuations in their velocities. For spherical particles this time $\tau_{B}$ is 
of the order of

$$
\tau_{\mathrm{B}}=\frac{m}{6 \pi \eta a}
$$

where $m$ is the mass of the colloidal particle. For times larger than $\tau_{\mathrm{B}}$ the mean-square displacement is given by

$$
\left\langle\Delta r^{2}(t)\right\rangle=6 D_{\mathrm{s}} t ; \quad t \gg \tau_{\mathrm{B}}
$$

where $D_{\mathrm{s}}$ is the self-diffusion coefficient.

In the case of non-interacting particles both the collective diffusion coefficient $D_{\text {c }}$ and the self-diffusion coefficient $D_{\mathrm{s}}$ are equal to $D_{0}$. For interacting particles this is no longer the case, however, and one has to distinguish between collective diffusion and self-diffusion. In addition, for self-diffusion one has to take into account that for times larger than $\tau_{1} \approx a^{2} / D$ the particle configuration can no longer be considered as effectively constant. As first noted by Pusey, ${ }^{5}$ this change in the particle configuration influences the mean-square displacement of the particles in such a way that it is now only linear for 'short' times $\tau_{\mathrm{B}} \ll t \ll \tau_{1}$ and 'long' times $t \gg \tau_{1}$, however with different values of the proportionality constant, i.e. different diffusion coefficients. Denoting the short- and long-time self-diffusion coefficients by $D_{\mathrm{s}}^{\text {short }}$ and $D_{\mathrm{s}}^{\text {long }}$ we may write

$$
\begin{array}{ll}
\left\langle\Delta r^{2}(t)\right\rangle=6 D_{\mathrm{s}}^{\text {short }} t ; & \tau_{\mathrm{B}} \ll t \ll \tau_{\mathrm{I}} \\
\left\langle\Delta r^{2}(t)\right\rangle=6 D_{\mathrm{s}}^{\text {long }} t ; & t \gg \tau_{\mathrm{I}} .
\end{array}
$$

In general $D_{\mathrm{s}}^{\text {long }}<D_{\mathrm{s}}^{\text {short }}$, indicating that the changes in the particle configuration on average give rise to a hindering of the motion of a diffusing particle.

In the case of polydisperse systems of colloidal particles one can distinguish between collective diffusion and exchange diffusion. ${ }^{6}$ Here exchange diffusion refers to the process where species of various kinds are exchanged at constant osmotic pressure. In case the particles in the polydisperse system differ only in marking then the exchange diffusion coefficient is precisely equal to $D_{\mathrm{s}}^{\text {long }}$.

Here we report dynamic light scattering (DLS) measurements of the short-time self-diffusion in a monodisperse system of large particles (radius $a \approx 400 \mathrm{~nm}$ ) and the collective and exchange diffusion in a polydisperse system of small particles (average radius $\vec{a}=10 \mathrm{~nm}$ ). Particularly the exchange diffusion appears to be a very sensitive function of concentration; for high concentrations (volume fraction $\phi=0.45$ ) the exchange diffusion process turns out to be of the order of $10^{3}$ times slower than collective diffusion.

This paper is organized as follows. In the next section we review briefly the connection between DLS and diffusion. In section 3 we present experimental DLS results on dispersions of large particles from which we obtain the short-time self-diffusion, and in section 4 DLS measurements on systems of small particles are analysed in terms of collective and exchange diffusion. The conclusions that can be drawn from this work are collected in section 5 .

\section{Dynamic Light Scattering and Diffusion ${ }^{1,2}$}

\subsection{Short-time Self-diffusion}

By DLS on a monodisperse suspension of colloidal particles one can determine the dynamic structure factor $F(\boldsymbol{q}, t)$ of the colloidal particles

$$
F(q, t)=\frac{1}{N}\left\langle\sum_{j, i=1}^{N} \exp \left\{\mathrm{i} \boldsymbol{q} \cdot\left[\boldsymbol{r}_{j}(t)-\boldsymbol{r}_{l}(0)\right]\right\}\right\rangle
$$


where $\boldsymbol{r}_{j}(t)$ is the position of the $j$ th particle at time $t$ and $\boldsymbol{q}$ is the scattering vector. From the time decay of this function information about the Brownian diffusion of the colloidal particles can be obtained. Specifically, one can define an effective diffusion coefficient as follows:

$$
D(q)=-\frac{\dot{F}(q, 0)}{q^{2} S(q)}
$$

where $S(q)=F(q, 0)$ is the static structure factor.

On the time-scale we are considering here, i.e. $t \gg \tau_{\mathrm{B}}$, the dynamics of the colloidal particles can be determined by the $N$-particle Smoluchowski equation: ${ }^{7}$

$$
\frac{\partial P\left(\boldsymbol{r}^{N}, t\right)}{\partial t}=\sum_{i, j=1}^{N} \frac{\partial}{\partial \boldsymbol{r}_{i}} \cdot \mathbf{D}_{i j}\left(\boldsymbol{r}^{N}\right) \cdot\left[\left(\frac{\partial U\left(\boldsymbol{r}^{N}\right)}{\partial \boldsymbol{r}_{j}}\right) \frac{P\left(\boldsymbol{r}^{N}, t\right)}{k T}+\frac{\partial P\left(\boldsymbol{r}^{N}, t\right)}{\partial \boldsymbol{r}_{j}}\right] .
$$

Here $P\left(\boldsymbol{r}^{N}, t\right)$ is the probability that the $N$ particles in the suspensions adopt the configuration $\boldsymbol{r}^{N}\left(\left\{\boldsymbol{r}_{1}, \boldsymbol{r}_{2}, \ldots, \boldsymbol{r}_{N}\right\}\right.$ being their cartesian coordinates) at time $t, U\left(\boldsymbol{r}^{N}\right)$ is the interparticle potential and $D_{i j}\left(\boldsymbol{r}^{N}\right)$ is the generalized diffusion tensor. The generalized Einstein result

$$
\mathbf{D}_{i j}=k T \mathbf{b}_{i j}
$$

relates $\mathbf{D}_{i j}$ to the mobility matrix $\boldsymbol{b}_{i j}$, which specifies a given particle's (coarse-grained) drift velocity $\boldsymbol{v}_{i}$ in response to forces $\boldsymbol{F}_{j}$ acting on the particles in the suspension

$$
\boldsymbol{v}_{i}=\sum_{j=1}^{N} \mathbf{b}_{i j} \cdot \boldsymbol{F}_{j}
$$

Using the $N$-particle Smoluchowski equation the time derivative appearing in eqn (8) can be calculated and one obtains

$$
D(q)=\frac{1}{N S(q) q^{2}} \sum_{j, l=1}^{N}\left\langle\boldsymbol{q} \cdot \mathbf{D}_{j l} \cdot \boldsymbol{q} \exp \left\{\mathrm{i} \boldsymbol{q} \cdot\left[\boldsymbol{r}_{j}(0)-\boldsymbol{r}_{l}(0)\right]\right\}\right\rangle .
$$

In the limit where qa is large, i.e. well beyond the oscillations in the structure factor, eqn (12) simplifies considerably because

$$
\lim _{q a \rightarrow \infty} S(q)=1
$$

and further the terms $j \neq l$ in the summation on the right-hand side of eqn (12) go to zero, leaving only the $N$ terms for which $j=l$. Thus we obtain

$$
\lim _{q a \rightarrow \infty} D(q)=\frac{\left\langle\boldsymbol{q} \cdot \mathbf{D}_{11} \cdot \boldsymbol{q}\right\rangle}{q^{2}} .
$$

This is precisely the short-time self-diffusion coefficient. Thus the short-time selfdiffusion coefficient can be measured by dynamic light scattering by using large particles $(a \geqslant 0.4 \mu \mathrm{m})$ such that one can study the temporal behaviour of $F(q, t)$ for values of $q$ beyond the oscillations in the structure factor, i.e. $q a \geqslant 12$.

$$
D_{\mathrm{s}}^{\text {short }}=\lim _{q a \rightarrow \infty}\left(-\frac{\dot{F}(q, 0)}{q^{2} S(q)}\right)=k T \frac{\left\langle\boldsymbol{q} \cdot \mathbf{b}_{11} \cdot \boldsymbol{q}\right\rangle}{q^{2}} .
$$

In order to calculate the short-time self-diffusion coefficient from eqn (15) one has to know the configuration-dependent mobility function $\mathbf{b}_{11}\left(\boldsymbol{r}^{N}\right)$. This quantity can be considered as a sum of one, two, three etc. particle contributions

$$
\mathbf{b}_{11}\left(\boldsymbol{r}^{N}\right)=\mathbf{b}_{11}^{(1)}+\sum_{j=2}^{N} \mathbf{b}_{11}^{(2)}\left(\boldsymbol{r}_{1}, \boldsymbol{r}_{j}\right)+\sum_{\substack{j, k=2 \\ j \neq k}}^{N} \mathbf{b}_{11}^{(3)}\left(\boldsymbol{r}_{1}, \boldsymbol{r}_{j}, \boldsymbol{r}_{k}\right)+\cdots
$$


Averaging the relevant two-particle mobility functions over the two-particle distribution function to (lowest) order in the density Batchelor ${ }^{8}$ obtained the first-order correction in $\phi$ to $D_{\mathrm{s}}^{\text {short: }}$

$$
D_{\mathrm{s}}^{\text {short }}=D_{0}(1-1.83 \phi) \text {. }
$$

The contribution in $\phi^{2}$ to $D_{\mathrm{s}}^{\text {short }}$ contains two distinct contributions: (i) the two-body hydrodynamic mobility function averaged over the second-order density contribution of the two-particle distribution and (ii) the three-body hydrodynamic mobility function averaged over the lowest-order density contribution of the three-particle distribution function. Using hydrodynamic mobility functions to order $(a / r)^{7}$ in the interparticle distance $r$, Beenakker and Mazur ${ }^{9}$ obtained

$$
D_{\mathrm{s}}^{\text {short }}=D_{0}\left(1-1.73 \phi-0.93 \phi^{2}+1.80 \phi^{2}\right)+\cdots
$$

where the first of the two terms of order $\phi^{2}$ is due to two-body hydrodynamic interactions, while the second results from three-body contributions. The difference in the contribution to order $\phi$ in eqn (17) and (18) is due to the fact that in obtaining eqn (17) accurate numerical values for the two-sphere hydrodynamic mobility functions were used, whereas eqn (18) was obtained using analytical results to order $(a / r)^{7}$. The higher terms in the density expansion are very difficult to calculate. Using a special summation technique Beenakker and Mazur ${ }^{10,11}$ succeeded in taking into account the many-body hydrodynamic interactions between an arbitrary number of spheres and obtained numerical values for the self-diffusion coefficient up to volume fractions of 0.45 .

\subsection{Exchange Diffusion}

In dynamic light scattering from a polydisperse colloidal system one can distinguish two kinds of fluctuations: (i) overall density fluctuations of the colloidal species and (ii) concentration fluctuations at constant overall density. Pusey et al. ${ }^{6}$ have argued that these fluctuations modes can be related to the fluctuating variables that appear in the work of Kirkwood and Goldberg. ${ }^{2}$ Adapted to the situation of a colloidal dispersion considered to be in dialytic equilibrium with the solvent (component 0 ) which is kept at constant chemical potential $\mu_{0}$ these variables can be written as

$$
\begin{aligned}
& \lambda_{1}=\sum_{k=1}^{r} \bar{V}_{k} \delta \rho_{k} \\
& \lambda_{p}=\frac{\delta \rho_{p}}{\rho_{p}}-\frac{\delta \rho_{1}}{\rho_{1}}, \quad p=2,3, \ldots, r .
\end{aligned}
$$

Here

$$
\bar{V}_{k}=\left(\frac{\partial V}{\partial N_{k}}\right)_{T, \Pi 1, N_{j \neq k}}
$$

where $V$ is the volume of the dispersion, $\Pi$ is the osmotic pressure, $N_{k}$ is the number of particles of the $k$ th species and $\rho_{k}$ is the number density of the $k$ th species. It is assumed that the system consists of $r$ colloidal species differing in size that can be considered as $r$ components.

It can be shown that fluctuations in the osmotic pressure are only caused by the variable $\lambda_{1}$. The fluctuation modes $\lambda_{2}, \lambda_{3}, \ldots, \lambda_{r}$ only give rise to fluctuations in the differences in the chemical potentials of the various species at constant osmotic pressure. Osmotic pressure gradients drive collective diffusion, whereas gradients in chemical potential differences cause exchange diffusion. The process of exchange diffusion, which involves the Brownian displacement of particles over distances larger than their own diameter, is closely related to long-time self-diffusion. 
The general polydisperse case is too complex for a detailed calculation of the amplitudes and decay times of the various modes; however, particularly at high concentrations, it may be argued that owing to the different relaxation rates there is little coupling between the collective diffusion mode and the exchange modes. Assuming this to be the case, the amplitude related to collective diffusion is proportional to $\left\langle\lambda_{1}^{2}\right\rangle$. Further, assuming that we are dealing with small hard spheres such that the scattering amplitude of the $k$ th species is proportional to $a_{k}^{3}$ and the thermodynamic quantities can be described by the Percus-Yevick theory, one obtains for the total scattering intensity ${ }^{6,13}$ (in the limits $q a \rightarrow 0$ )

$$
A_{\text {tot }}=L\left(1-\xi_{3}\right)^{2}\left[\xi_{6}-\frac{6 \xi_{4} \xi_{5}}{\left(1+2 \xi_{3}\right)}+\frac{9 \xi_{4}^{3}}{\left(1+2 \xi_{3}\right)^{2}}\right] \text {. }
$$

Here $L$ is a proportionality constant that does not concern us here and

$$
\xi_{\nu}=\frac{\pi}{6} \sum_{k=1}^{r} \rho_{k}\left(2 a_{k}\right)^{\nu}
$$

Under the conditions for which the total intensity is given by eqn (22) one obtains for the light scattering intensity associated with the fluctuations in the collective mode $\lambda_{1}$ the following results: ${ }^{6,13}$

$$
A_{+}=\frac{L \xi_{3}^{2}\left(1-\xi_{3}\right)^{4}}{\xi_{0}\left(1-\xi_{3}\right)^{2}+6 \xi_{1} \xi_{2}\left(1-\xi_{3}\right)+9 \xi_{2}^{3}} .
$$

The remaining light scattering contribution, $A_{-}=\left(A_{\text {tot }}-A_{+}\right)$, will relax according to the process of exchange diffusion. The relative weights of $\boldsymbol{A}_{+}$and $\boldsymbol{A}_{-}$are mainly determined by the standard deviation of the size distribution:

$$
\sigma=\left[\left(\overline{a^{2}}-\bar{a}^{2}\right) / \bar{a}^{2}\right]^{1 / 2}
$$

and are relatively insensitive to the type of distribution. For narrow distributions one obtains $^{6}$

$$
\frac{A_{+}}{A_{\mathrm{tot}}}=1-\frac{A_{-}}{A_{\mathrm{tot}}}=\frac{1}{\left[1+\frac{3 \sigma^{2} \phi(4-\phi)}{(1-\phi)^{2}}\right]\left[1+\frac{3 \sigma^{2}\left(7 \phi^{2}+8 \phi+3\right)}{(1+2 \phi)^{2}}\right]}
$$

where $\phi=\xi_{3}$ is the total volume fraction.

\section{Dynamic Light Scattering in Dispersions of Large Particles}

Short-time self-diffusion coefficients were measured in two dispersions, both containing silica particles of $440 \mathrm{~nm}$ radius. The silica particles in the first system were coated with $\gamma$-methacryloxypropyltrimethoxysilane (TPM), whereas the particles in the second system were first coated with TPM, followed by a second coating reaction with trimethylhydroxysilane (TMHS). Both types of particles are sterically stabilized, but, possibly because not all silanol groups have reacted with TPM, the first type of particle still carries a negative charge, as indicated by the fact that it shows electrophoretic mobility. The remaining silanol groups can be blocked by a second coating reaction with a small molecule (in this case TMHS). The particles of the second system turned out to be uncharged in tetrahydrofurfuryl alcohol (THFA), the solvent used in the experiments (see below).

To avoid multiple scattering, which would complicate the results of DLS measurements, one has to select a solvent with a refractive index close to that of the particles. Furthermore, the particles have to be stable in this solvent. It appears that THFA satisfies both requirements. The optical matching point was situated between 30 and $35^{\circ} \mathrm{C}$. 


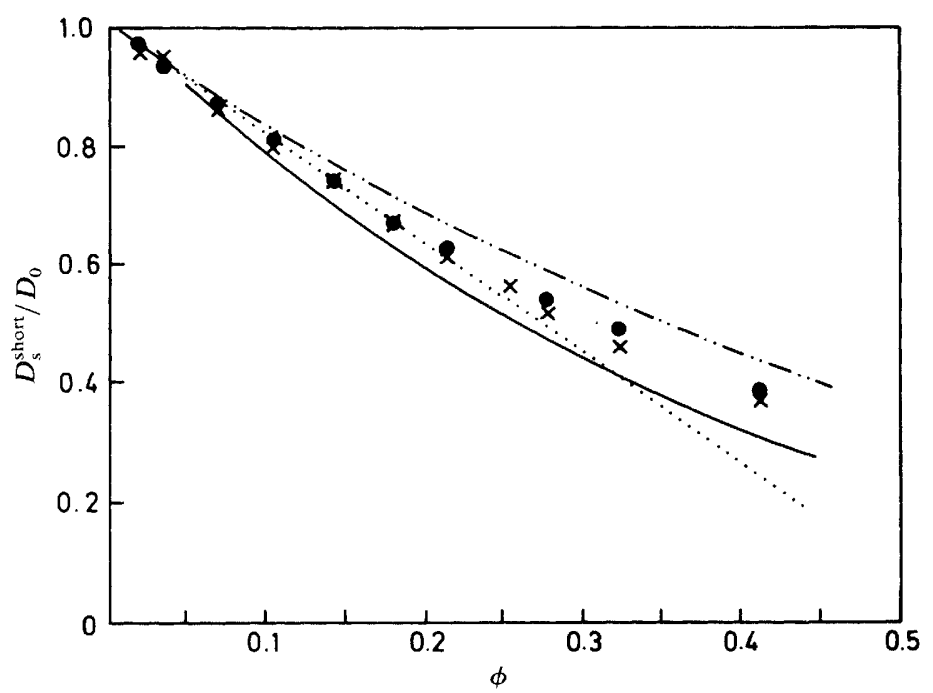

Fig. 1. Normalised short-time self-diffusion coefficients for double-coated silica particles at $30^{\circ} \mathrm{C}$ $(\times)$ and $35^{\circ} \mathrm{C}(\mathbf{O}) .(\cdots)$ is the $0(\phi)$ result [eqn $(17)$ ], $(-\cdots-)$ is the $0\left(\phi^{2}\right)$ result [eqn $(18)$ ] and $(-)$ represents the many-body result of Beenakker and Mazur [ref. (10) and (11)].

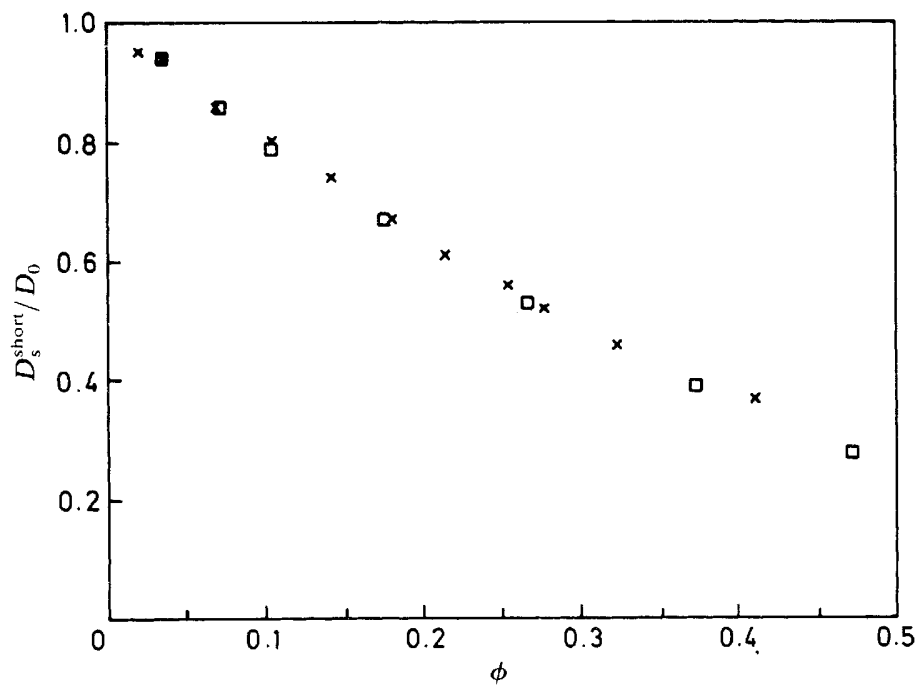

Fig. 2. Normalised short-time self-diffusion coefficients for single- $(\square)$ and double-coated $(\times)$ silica particles at $30^{\circ} \mathrm{C}$.

The value of $D_{0}$ was obtained both by extrapolation of data taken at low concentrations in THFA as well as from measurements on dilute systems to which a trace amount of toluene had been added to enhance the particle scattering and thereby the signal-tonoise ratio of the measured autocorrelation function.

In fig. 1 we compare the experimental data from the second system (double-coated silica particles) with the various theoretical results described in section 2.1. Volume fractions were calculated from weight fractions using the densities of the silica particles 
and solvent, 1.58 and $1.04 \mathrm{~g} \mathrm{~cm}^{-3}$, respectively. We note that the first-order correction in $\phi$ describes the data surprisingly well up to $\phi \approx 0.20$. For the high concentrations the calculations of Beenakker and Mazur ${ }^{10,11}$ that take into account many-body hydrodynamics come close to the experimental points. Similar conclusions have already been reached by Pusey and van Megen ${ }^{14}$ for the short-time self-diffusion of sterically stabilized poly(methyl methacrylate) (PMMA) particles.

In fig. 2 we compare data obtained from the charged (single-coated) system with data obtained from the uncharged (double-coated) system. There appears to be remarkably little difference between them. This indicates that the short-time self-diffusion is mainly determined by hydrodynamic interactions and is apparently affected little by direct particle interactions.

\section{Dynamic Light Scattering in Polydisperse Systems of Small Particles}

In order to measure the exchange diffusion and mode amplitudes as discussed in section 2.2. We performed DLS measurements on polydisperse systems of small silica particles, sterically stabilized by a coating of stearyl chains, dispersed in cyclohexane. These particles are known to behave like hard spheres. ${ }^{15}$

We compare the data obtained from two dispersions differing only in size and degree of polydispersity of the particles. These systems will hereafter be referred to as systems I and II. The two systems were characterized by DLS measurements on dilute systems. From a second-order cumulant expansion an apparent diffusion coefficient (or apparent radius) and polydispersity index were obtained. From these the number-averaged mean radius $\bar{a}$ and standard deviation $\sigma$ were calculated. In this way we found for system I the values $\vec{a}=10 \mathrm{~nm}$ and $\sigma=0.3$ and for system II $\bar{a}=21 \mathrm{~nm}$ and $\sigma=0.2$. These values are consistent with the results obtained from the apparent radii of gyration as measured by static light scattering. As far as the number-averaged mean radius is concerned the above values are also consistent with the values obtained by electron microscopy $(\bar{a}=9 \pm 1 \mathrm{~nm}$ for system I and $\bar{a}=23 \pm 3 \mathrm{~nm}$ for system II). On the other hand, we found that the standard deviations as obtained from electron microscopy and small-angle neutron scattering are significantly lower $(\sigma=0.10-0.15)$ than the values obtained from DLS. These differences may possibly be attributed to the presence of clusters of particles in the dispersion. This phenomenon would have a pronounced effect on scattering at low $q$ (light scattering), but rather little influence on scattering at high $q$ (small-angle neutron scattering) and electron microscopy.

DLS measurements were made up to volume fractions of 0.55 . The volume fractions were calculated from weight fractions using the densities of the silica particles and the solvent, 1.58 and $0.774 \mathrm{~g} \mathrm{~cm}^{-3}$, respectively. It turns out that with increasing concentration the measured electric field autocorrelation function definitely consists of a fastand a slow-decaying part. The amplitudes associated with these parts can be determined reasonably well from these measurements and are given in fig. 3 . The decay times associated with the slow and the fast parts differ considerably (see table 1).

Because the slow decay was definitely not single-exponential, we give in table 1 a range of decay times, expressed as diffusion coefficients, instead of one single exchange diffusion coefficient. The lower values represent the overall decay of the slow part of the field autocorrelation function, whereas the upper values were determined from the initial part of the slow decay, where in any case the fast mode has effectively fully decayed. The amplitudes displayed in fig. 3 correspond to the latter fitting procedure. The decay time of the slow part increases rapidly with increasing concentration, whereas the fast mode shows little concentration dependence.

These results are in qualitative agreement with the available theory. ${ }^{6,13}$ However, there are some differences. First, we have observed that not only is the slowly relaxing part multi-exponential (as was expected from theory) but it appears that the fast decay 


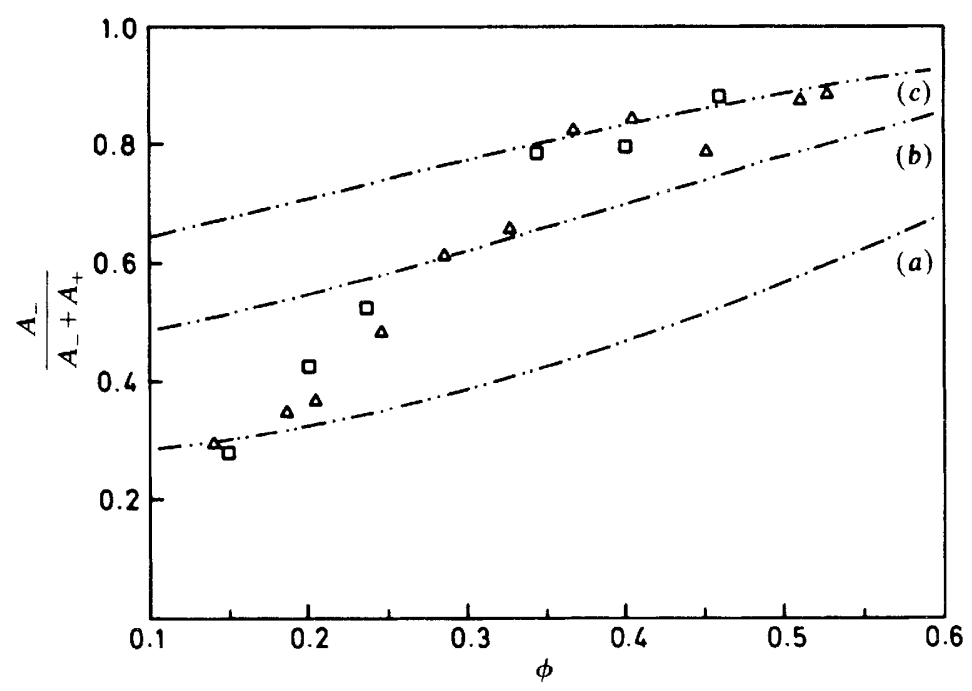

Fig. 3. Relative amplitudes of the slow mode as a function of the total volume fraction $\phi$ for system I $(\square)$ and for system II $(\Delta)$. The theoretical curves (-..-) were calculated using eqn (25): (a) $\sigma=2,(b) \sigma=3$, (c) $\sigma=4$.

Table 1. Values of the decay constant of the fast mode $\left(D_{+}\right)$and the range of decay constants of the slow modes $\left(D_{-}\right)$for system I

\begin{tabular}{cccc}
\hline & & \multicolumn{2}{c}{$D_{-} / 10^{-12} \mathrm{~m}^{2} \mathrm{~s}^{-1}$} \\
\cline { 3 - 4 }$\phi$ & $D_{+} / 10^{-12} \mathrm{~m}^{2} \mathrm{~s}^{-1}$ & lower value & upper value \\
\hline 0.15 & 21.3 & 1 & 3 \\
0.20 & 22.3 & 0.45 & 0.9 \\
0.24 & 23.0 & 0.3 & 0.6 \\
0.34 & 27.7 & 0.045 & 0.12 \\
0.40 & 26.5 & 0.016 & 0.08 \\
0.46 & 31 & 0.001 & $(<0.08)$ \\
\hline
\end{tabular}

part is also not single-exponential. Furthermore, the dependence on overall concentration of the relative amplitudes is more pronounced than predicted by theory for both systems studied. A possible explanation for this may be that the scattering power polydispersity is not only caused by size differences but also by differences in the refractive index of the particles.

\section{Conclusions}

With DLS measurements various Brownian diffusion characteristics of interacting colloidal particles can be investigated. Monodisperse dispersions of large particles allow us to probe the short-time self-diffusion. It appears that the short-time self-diffusion is not very sensitive to direct particle interactions. Polydisperse dispersions of small particles allow us to probe long-time diffusive behaviour. For concentrated dispersions of small particles, polydisperse in size, the measured electric field autocorrelation function is composed of two groups of modes with well separated decay times. The fast mode may be associated with collective diffusion and the slow modes with exchange 
diffusion. Theoretically, this mode-decoupling is only expected for relatively high volume fractions. In view of this, and because of the uncertainty in the degree of the polydispersity, it is difficult to say whether the deviations of the measured modeamplitudes from the theoretical predictions are of great significance.

The collective diffusion shows little concentration dependence, whereas both shorttime self-diffusion and exchange diffusion become slower with increasing concentration. However, whereas for volume fractions of 0.45 short-time self-diffusion becomes roughly a factor of 5 slower compared to the limit of infinite dilution, exchange diffusion becomes up to 1000 times slower, indicating that diffusion over distances larger than the diameter of the particles becomes extremely slow in concentrated dispersions. This may explain why dispersions of hard spheres at high concentrations so readily form a glass. ${ }^{16,17}$

The silica spheres coated with TPM and TMHS were synthesized by Mr A. P. Philipse and $\mathrm{Mr} \mathrm{V}$. de Leeuw, and the silica spheres coated with stearyl chains (system II) were synthesized by $\mathrm{Mr} \mathrm{G}$. Geels. We are grateful to them for providing these systems. $\mathrm{Mr}$ $\mathrm{S}$. Coenen is thanked for assistance with the DLS measurements. We thank Mrs M. Uit de Bulten for typing the manuscript. This work is part of the research program of the Foundation for Fundamental Research of Matter (FOM) with financial support from the Netherlands Organisation for Pure Research (ZWO).

\section{References}

1 P. N. Pusey and R. J. A. Tough, Dynamic Light Scattering Applications of Photon Correlation Spectroscopy, ed. R. Pecora (Plenum Press, New York, 1985), pp. 85-179.

2 J. M. Rallison and P. J. Hinch, J. Fluid Mech., 1986, 167, 131.

3 P. Mazur, Can. J. Phys., 1985, 63, 24.

4 A. Einstein, Investigations on the Theory of Brownian Movement (Dover Publications, New York, 1956).

5 P. N. Pusey, J. Phys. A, 1975, 8, 1433.

6 P. N. Pusey, H. M. Fijnaut and A. Vrij, J. Chem. Phys., 1982, 77, 4270.

7 R. J. A. Tough, P. N. Pusey, H. N. W. Lekkerkerker and C. Van den Broeck, Mol. Phys., 1986, $59,595$.

8 G. K. Batchelor, J. Fluid Mech., 1976, 74, 1.

9 C. W. J. Beenakker and P. Mazur, Phys. Lett. A, 1982, 91, 290.

10 C. W. J. Beenakker and P. Mazur, Phys. Lett. A, 1983, 98, 22.

11 C. W. J. Beenakker and P. Mazur, Physica, 1984, 126A, 349.

12 J. G. Kirkwood and R. J. Goldberg, J. Chem. Phys., 1950, 18, 54.

13 A. Vrij, J. Colloid Interface Sci., 1982, 90, 110.

14 P. N. Pusey and W. Van Megen, J. Phys. (Paris), 1983, 44, 258.

15 A. Vrij, J. W. Jansen, J. K. G. Dhont, C. Pathmamanoharan, M. M. Kops-Werkhoven and H. M. Fijnaut, Faraday Discuss. Chem. Soc., 1983, 76, 19.

16 C. G. de Kruif, P. W. Rouw, J. W. Jansen and A. Vrij, J. Phys. (Paris), 1985, 46, C3-295.

17 P. N. Pusey and W. Van Megen, Nature (London), 1986, 320, 340. 